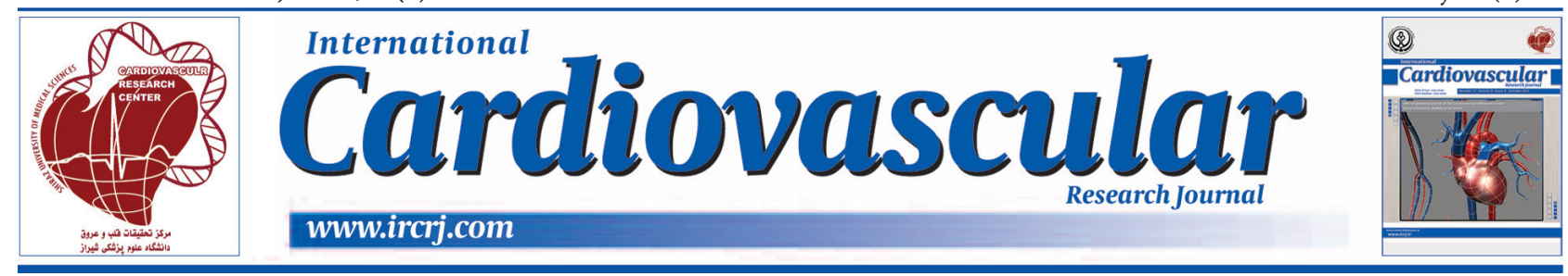

\title{
The Relationship between Tpeak-end Interval Duration and Tpeak-end/QT Ratio, and Arrhythmias in Patients with Coronary Slow Flow
}

\author{
Fatih Sen ${ }^{1}$, Samet Yılmaz ${ }^{2,}$, Firat Özcan ${ }^{1}$, Özcan Özeke ${ }^{1}$, Serkan Çay ${ }^{1}$, Serkan Topaloğlu ${ }^{1}$, Dursun \\ Aras ${ }^{1}$, Sinan Aydoğdu ${ }^{1}$
}

\footnotetext{
${ }^{1}$ Turkey Yuksek Ihtisas, Education and Research Hospital, Cardiology Clinic, Ankara, Turkey

${ }^{2}$ Yozgat State Hospital, Cardiology Clinic, Yozgat, Turkey
}

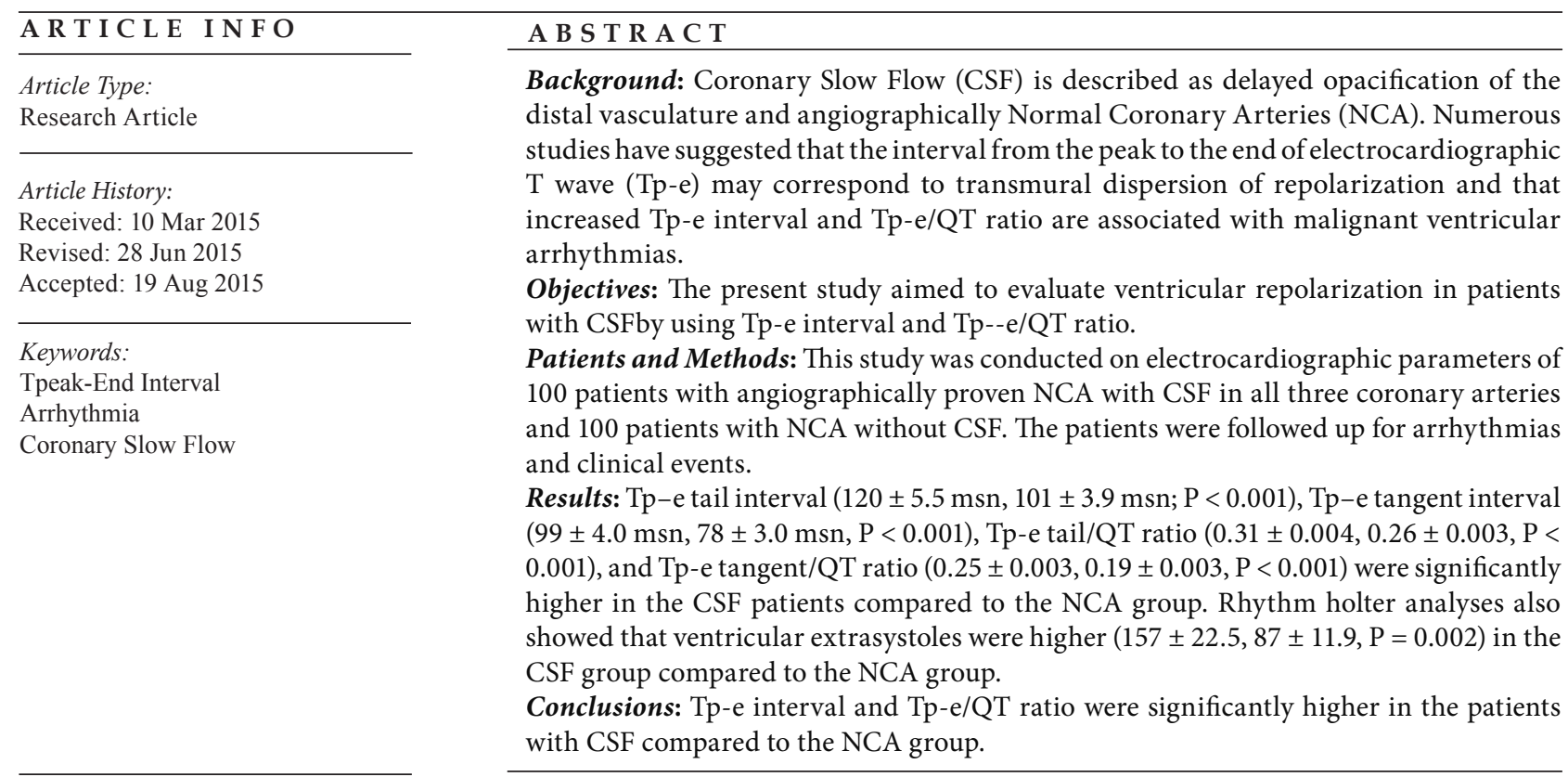

Implication for health policy/practice/research/medical education:

$\mathrm{Tp}$-e interval is an important electrocardiographic parameter to predict arrhythmias in patients with coronary slow flow.

\section{Background}

Coronary Slow Flow (CSF) is an angiographic finding characterized by delayed opacification of coronary arteries in the absence of epicardial occlusive disease. CSF is not a rare finding in patients undergoing routine coronary angiography and its prevalence has been reported to be $5.5 \%$ (1). Rather than representing a simple angiographic curiosity, CSF has direct clinical implications as it has been linked to clinical manifestations of myocardial ischemia, ${ }^{*}$ Corresponding author: Samet Yilmaz, Yozgat State Hospital Cardiology
Clinic, 66100, Yozgat, Turkey. Tel: 0090-5073055883,

E-mail: sametyilmazmd@gmail.com life threatening arrhythmias, sudden cardiac death, and recurrent acute coronary syndromes (2-5).

$\mathrm{T}$ wave is the electrocardiographic manifestation of ventricular repolarization. Recent experimental evidences have suggested that the interval from the peak to the end of the $\mathrm{T}$ wave ( $\mathrm{Tp}$-e interval) corresponds to dispersion of ventricular repolarization (6-8). Increased Tp-e interval might be a useful index to predict ventricular tachyarrhythmias and cardiovascular mortality (9-11). Recently, a new index, Tp-e/QT ratio, has been suggested to be a more accurate measure for dispersion of ventricular repolarization compared to QT, corrected QT (QTc), and 
Tp-e intervals that are independent from alterations in heart rate $(12,13)$.

\section{Objectives}

The present study aims at evaluating the effects of CSF on Tp-e interval and Tp-e/QT ratio as a possible indicator of increased risk of ventricular arrhythmias and sudden cardiac death.

\section{Patients and Methods}

The study population included 100 patients with angiographically proven Normal Coronary Arteries (NCA) and CSF in all the three coronary vessels and 100 patients with angiographically proven NCA without associated CSF. Patients with left ventricular dysfunction, echocardiographically proven left ventricular hypertrophy, Electrocardiography (ECG) with atrial fibrillation, bundle branch block, and evidence of any other intraventricular conduction defects or electrolyte abnormalities were excluded from the study. In order to detect any possible arrhythmia, 24-hour rhythm holter monitoring was performed for all the patients within 48 hours after coronary angiography. The patients were followed by routine outpatient clinic visits. It should be mentioned that written informed consents were obtained from all the participants and the study protocol was approved by the local Ethics Committee.

\subsection{Documentation of Coronary Slow Flow}

After coronary angiography, the patients' coronary flow rates were documented by Thrombolysis in Myocardial Infarction (TIMI) frame count. The TIMI frame count method is a simple, reproducible, objective, and quantitative index of coronary flow velocity (14). The TIMI frame count was determined for each major coronary artery in each patient according to the method that was first described by Gibson et al. (14). Briefly, the number of cineangiographic frames, recorded at 30 frames per second, required for the leading edge of the column of radiographic contrast to reach a predetermined landmark was determined. The first frame was defined as the frame in which the concentrated dye occupied the full width of the proximal coronary artery lumen, touching both borders of the lumen, and in forward motion proceeded down the artery. Besides, the final frame was designated when the leading edge of the contrast column initially arrived at the distal landmark. In the Left Anterior Descending (LAD) coronary artery, the used landmark was the most distal branch nearest the apex of the left ventricle, commonly referred to as the "pitchfork" or the "whale's tail". LAD coronary artery is usually longer than the other major coronary arteries; therefore, the TIMI frame count is often higher for this vessel (15). To obtain a corrected TIMI frame count for LAD coronary artery, the TIMI frame count was divided by 1.7 (14).

The Right Coronary Artery (RCA) distal landmark is the first branch of the posteriolateral RCA after the origin of the posterior descending artery, regardless of the size of this branch. The branch of the Left Circumflex (LCx) artery that encompassed the greatest total distance traveled by contrast was used to define the distal landmark of the LCX artery. The TIMI frame count was assessed in right anterior oblique projection with caudal angulation in the LAD and LCx arteries and in left anterior oblique projection with cranial angulation in the RCA.

\subsection{Electrocardiography}

All standard 12-lead ECGs were obtained at rest in the supine position simultaneously using a recorder (Nihon Kohden, Tokyo, Japan) set at $50 \mathrm{~mm} / \mathrm{s}$ paper speed and 1 $\mathrm{mV} / \mathrm{cm}$ standardization. Assessments of the ECGs were done by 2 cardiologists to diminish measurement errors. Lead V5 was selected for analysis. QRS interval duration and Tp-e interval duration measurements were made on lead V5. When the T wave in V5 was $<1 \mathrm{~mm}$ in height or was obscured by a large U wave, lead II was used. QT interval was measured in a single complex from the beginning of the QRS complex to the end of the T wave. QTc interval was the QT interval corrected with the Bazett's formula (QT/ $\sqrt{ } R R)$ (16). Besides, Tp-e was defined as the interval from the peak of a positive $T$ wave or the nadir of a negative $\mathrm{T}$ wave to the end of the $\mathrm{T}$ wave in the tail method (17). In the tangent method, Tp-e (Tp-e tangent) was defined as the time from the peak of the $\mathrm{T}$ wave to the intersection between the tangent line and the isoelectric line $(9,18)$ (Figure 1). Although there is no consensus regarding heart rate correction of $\mathrm{Tp}-\mathrm{e}$, Bazett's formula (n/ $\sqrt{ } \mathrm{RR}$ ) was applied to $\mathrm{Tp}-\mathrm{e}$ tail and $\mathrm{Tp}-\mathrm{e}$ tangent intervals to determine $\mathrm{cTp}-\mathrm{e}$ tail and cTp-e tangent, respectively. Tp-e/QT ratio was also calculated for all the ECGs. Although measurements were performed independently by two blinded physicians, when measurements were not identical, the mean values were calculated. Additionally, in case the values differed by $>10$ $\mathrm{ms}$, the measurements were adjudicated by a third reviewer.

\subsection{Statistical Analysis}

Continuous variables were expressed as mean \pm SEM, while categorical ones were expressed as percentage. The two groups were compared regarding categorical and continuous variables using chi-square test and unpaired t-test, respectively. In addition, the correlation between Tp-e and TIMI frame count was assessed by Pearson's correlation test. $\mathrm{P}$ value $<0.05$ was considered to be statistically significant.

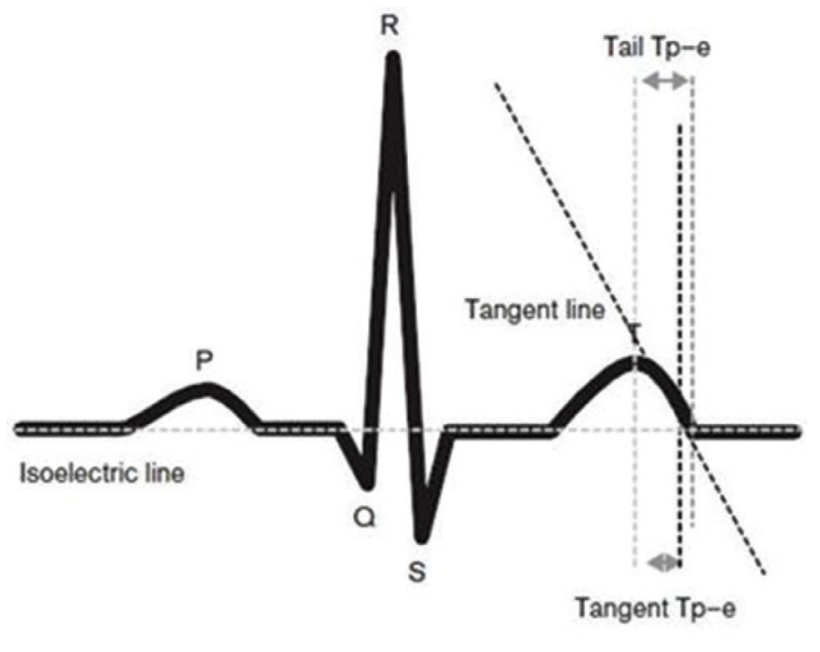

Figure 1. Schematic Presentation of Tp-e Measurement 


\section{Results}

The baseline characteristics of the study population have been presented in Table 1. Accordingly, no statistically significant differences were found between the two groups with respect to age, gender, and presence of hypertension and dyslipidemia $(\mathrm{P}>0.05)$. However, compared to the NCA group, the number of patients with diabetes mellitus, cigarette smoking, and family history of atherosclerosis was significantly higher in the CSF group. TIMI frame counts of the CSF group were also significantly higher than those of the NCA group for all the three coronary arteries $(\mathrm{P}<$ 0.001 for all, Table 2).

Comparison of the ECG parameters has been presented in Table 3. Although the two groups were not different regarding QRS duration and QT and QTc intervals, Tp-e tail interval $(120 \pm 5.5 \mathrm{msn}, 101 \pm 3.9 \mathrm{msn} ; \mathrm{P}<0.001)$ and Tp-e tangent interval $(99 \pm 4.0 \mathrm{msn}, 78 \pm 3.0 \mathrm{msn}, \mathrm{P}<0.001)$ were significantly prolonged in the CSF group compared to the NCA group. Also, Tp-e tail/QT ratio $(0.31 \pm 0.004$,
$0.26 \pm 0.003, \mathrm{P}<0.001)$ and Tp-e tangent/QT ratio $(0.25 \pm$ $0.003,0.19 \pm 0.003, \mathrm{P}<0.001)$ were significantly higher in the CSF patients compared to the NCA group. In addition, a significant correlation was observed between Tp-e tail and TIMI frame counts ( $\mathrm{P}<0.001$, Table 4$)$.

The mean duration of coronary angiography follow-up was $17 \pm 1.2$ months. The results of rhythm holter have been shown in Table 5. Accordingly, there were $157 \pm$ 22.5 and $87 \pm 11.9$ Ventricular Extrasystoles (VES) in the CSF and NCA groups, respectively $(\mathrm{P}=0.002)$. Moreover, there were 6 case of non-sustained Ventricular Tachycardia (VT) in the CSF group (4 patients had 1 episode and 1 patient had 2 episodes) and 2 in the normal coronary flow group (2 patients had 1 episode) $(\mathrm{P}=0.003)$. Furthermore, a significant positive correlation was found between the number of VES and QTC interval $(r=0.195, P=0.024)$.

\section{Discussion}

The present study showed that Tp-e interval and Tp-e/QT

\begin{tabular}{|c|c|c|c|}
\hline $\mathrm{N}=200$ & $\operatorname{CSF}(n=100)$ & NCA $(n=100)$ & $P$ value \\
\hline Age (year \pm SEM) & $53.4 \pm 1$ & $52.4 \pm 0.86$ & 0.431 \\
\hline Male/female & $49 / 51$ & $47 / 53$ & 0.922 \\
\hline Hypertension (\%) & 62 & 48 & 0.073 \\
\hline Diabetes mellitus (\%) & 38 & 25 & 0.048 \\
\hline Dyslipidemia (\%) & 18 & 17 & 0.854 \\
\hline Family history $(\%)^{\star}$ & 56 & 39 & 0.016 \\
\hline Smoking (\%) & 80 & 51 & $<0.001$ \\
\hline Systolic blood pressure $(\mathrm{mmHg})$ & $128 \pm 1.95$ & $128 \pm 1.7$ & 0.985 \\
\hline Diastolic blood pressure (mmHg) & $79.8 \pm 1.3$ & $78.5 \pm 1.2$ & 0.626 \\
\hline Body mass index $(\mathrm{kg} / \mathrm{m} 2)$ & $28.4 \pm 0.37$ & $27.8 \pm 0.29$ & 0.677 \\
\hline Ejection fraction (\%) & $63 \pm 0.5$ & $62 \pm 0.7$ & 0.888 \\
\hline
\end{tabular}

*Family history: First-degree relatives with coronary artery disease aging $<45$ years in men and $<55$ years in women.

\begin{tabular}{llll}
\hline Table 2. Thrombolysis in Myocardial Infarction Frame Counts of the Patients and the Control Subjects & \\
\hline N = 200 & CSF $(\mathbf{n}=\mathbf{1 0 0})$ & NCA $(\mathbf{n}=\mathbf{1 0 0})$ & P value \\
\hline LAD & $48.1 \pm 1.13$ & $22.9 \pm 0.496$ & $<0.001$ \\
LCx & $34.4 \pm 0.96$ & $19.6 \pm 0.46$ & $<0.001$ \\
RCA & $26.4 \pm 0.92$ & $12.9 \pm 0.38$ & $<0.001$ \\
\hline
\end{tabular}

Abbreviations: LAD, left anterior descending artery; LCx, left circumflex artery; RCA, right coronary artery

\begin{tabular}{llll}
\hline \multicolumn{1}{l}{ Table 3. Comparison of ECG Data Between the Patients } & & \\
\hline & CSF $(\mathbf{n}=\mathbf{1 0 0})$ & NCA (n = 100) & P value \\
\hline Heart rate (beats/min) & $74 \pm 0.6$ & $72 \pm 0.5$ & 0.711 \\
Tp-e tail (ms) & $120 \pm 5.5$ & $101 \pm 3.9$ & $<0.001$ \\
Tp-e tangent (ms) & $99 \pm 4.0$ & $78 \pm 3.0$ & $<0.001$ \\
QRS duration (ms) & $101.5 \pm 12.1$ & $96.8 \pm 11.9$ & 0.122 \\
QT interval (ms) & $392.1 \pm 14.9$ & $390.3 \pm 4.32$ & 0.754 \\
Corrected QT interval (ms) & $419.1 \pm 14.4$ & $415 \pm 15.1$ & 0.524 \\
Tp-e tail/QT & $0.31 \pm 0.004$ & $0.26 \pm 0.003$ & $<0.001$ \\
Tp-e tangent/QT & $0.25 \pm 0.003$ & $0.19 \pm 0.003$ & $<0.001$ \\
\hline
\end{tabular}

\begin{tabular}{llr}
\hline Table 4. The Correlation between Tp-e tail Duration and Thrombolysis in Myocardial Infarction Frame Count & \\
\hline $\mathbf{N}=\mathbf{2 0 0}$ & Pearson's Correlation & P value \\
\hline LAD & 0.62 & $<0.001$ \\
CX & 0.58 & $<0.001$ \\
RCA & 0.64 & $<0.001$ \\
\hline
\end{tabular}

LAD, left anterior descending artery; LCx, left circumflex artery; RCA, right coronary artery 


\begin{tabular}{llll}
\hline \multicolumn{2}{l}{ Table 5. The Results of the Patients' 24-hour ECG Holter Monitoring } & & \\
\hline $\mathbf{N = 2 0 0}$ & CSF $(\mathbf{n}=\mathbf{1 0 0})$ & NCA $(\mathbf{n}=\mathbf{1 0 0})$ & P value \\
\hline Mean heart rate & $85 \pm 2.1$ & $76 \pm 1.9$ & 0.44 \\
Minimum heart rate & $46 \pm 0.5$ & $48 \pm 0.5$ & 0.82 \\
Maximum heart rate & $145 \pm 2.2$ & $128 \pm 2.0$ & 0.25 \\
Ventricular extra systole & $157 \pm 22.5$ & $87 \pm 11.9$ & 0.003 \\
Episodes of non-sustained VT & 6 & 2 & 0.002 \\
\hline
\end{tabular}

${ }^{*}$ Non-sustained VT: VT that lasts no longer than 30 seconds.

ratio were prolonged in the patients with CSF compared to those with normal coronary flow.

There has been considerable interest in the association between repolarization patterns and their electrocardiographic correlates and risk of arrhythmia. Tp-e interval may be a measure of transmural dispersion of recovery properties and many studies have shown that it is associated with arrhythmias. In addition, subsequent retrospective analyses in specific human populations, including those with acquired long QT syndrome, Brugada syndrome, and hypertrophic cardiomyopathy, showed Tp-e to be associated with torsades de pointes, appropriate Implantable Cardioverter Defibrillator (ICD) therapy, and sudden cardiac death, respectively $(9,18)$. Correspondingly, holter analysis of our patients showed that number of VES and non-sustained VT were significantly higher in the patients with CSF compared to those with NCA. The patients with CSF also had more cardiovascular risk factors, such as diabetes, smoking, and positive family history. These factors might have also caused VES via development of atherosclerosis. However, all the patients underwent angiography and they did not have obstructive Coronary Artery Disease (CAD). In a small case-control study on a heterogeneous population of patients undergoing Electrophysiology Study (EPS), Watanabe et al. showed that inducibility of VT at EPS correlated to longer corrected Tp-e duration (13). In patients undergoing percutaneous coronary intervention for STelevation myocardial infarction, a longer pre-PCI (but not post- PCI) Tp-e predicted all-cause one-year mortality (12). In patients with left ventricular systolic dysfunction and ICD therapy, Tp-e predicted both appropriate ICD therapy for ventricular tachyarrhythmia and overall mortality, even after correction for other independent predictors (14).

Slow runoff dye in the coronary arteries during selective coronary angiography is known as slow coronary artery flow (19). Since its original description by Tambe et al. in 1972, only scarce attention has been paid to this phenomenon and, consequently, its clinical significance has remained unclear. Some authors suggested that abnormal increase of small vessel resistance was the cause of the slow dye progression in selective coronary angiography (20-22). It is believed to represent coronary microvascular dysfunction. Histopathological studies have also revealed the existence of fibromuscular hyperplasia, medial hypertrophy, myointimal proliferation, and endothelial degeneration in microvascular circulation from the right ventricular biopsy specimens of patients with slow coronary artery flow (21). It has been suggested that these histopathological changes lead to an abnormally increased microvascular tone and, as a result, most patients with CSF complain mainly about typical cangina pectoris. Yaymaci et al. documented the presence of myocardial ischemia in $83.4 \%$ of these patients with positive scintigraphic findings (23). Furthermore, some researchers have shown exercise-induced ST-segment depression in patients with CSF without obstructive CAD $(23,24)$. Therefore, increased Tp-e durations in patients with CSF might be attributed to impaired coronary blood flow, which might result from microvascular dysfunction. Coronary insufficiency caused by impaired coronary blood flow seems to be the most valuable explanation for increased left ventricular repolarization heterogeneity in patients with CSF.

In conclusion, the current study results revealed that $\mathrm{Tp}$-e and Tp-e/QT, indicating increased risk of ventricular arrhythmias and cardiovascular mortality, were significantly higher in the patients with CSF. However, further prospective studies should be carried out to establish the significance of Tp-e and Tp-e/QT, as risk factors of ventricular arrhythmias and the subsequent sudden cardiac death, in patients with CSF.

\section{Acknowledgements}

There is no acknowledgement.

\section{Authors' Contribution}

Study concept and design: Fatih Sen, SametYilmaz; Acquisition of data: Fatih Sen; Analysis and interpretation of data: Samet Yilmaz; Drafting of the manuscript: Firat Ozcan; Critical revision of the manuscript for important intellectual content: Ozcan Ozeke; Statistical analysis: Dursun Aras, Administrative, technical, and material support: Serkan Topaloglu; Study supervision: Sinan Aydoğdu

\section{Financial disclosure}

There is no financial disclosure.

\section{Funding/Support}

There is no funding/support.

\section{References}

1. Hawkins BM, Stavrakis S, Rousan TA, Abu-Fadel M, Schechter E. Coronary slow flow--prevalence and clinical correlations. Circ J. 2012;76(4):936-42.

2. Cutri N, Zeitz C, Kucia AM, Beltrame JF. ST/T wave changes during acute coronary syndrome presentation in patients with the coronary slow flow phenomenon. Int J Cardiol. 2011;146(3):457-8.

3. Horjeti B, Goda A. Acute ischemia manifestation in a patient with coronary slow flow phenomenon. J Electrocardiol. 2012;45(3):277-9.

4. Saya S, Hennebry TA, Lozano P, Lazzara R, Schechter E. Coronary slow flow phenomenon and risk for sudden cardiac death due to ventricular arrhythmias: a case report and review of literature. Clin Cardiol. 2008;31(8):352-5.

5. Wozakowska-Kaplon B, Niedziela J, Krzyzak P, Stec S. Clinical manifestations of slow coronary flow from acute coronary syndrome to serious arrhythmias. Cardiol J. 2009;16(5):462-8. 
6. Antzelevitch C. T peak-Tend interval as an index of transmural dispersion of repolarization. Eur J Clin Invest. 2001;31(7):555-7.

7. Antzelevitch C. Role of spatial dispersion of repolarization in inherited and acquired sudden cardiac death syndromes. Am J Physiol Heart Circ Physiol. 2007;293(4):H2024-38.

8. Antzelevitch C. Heterogeneity and cardiac arrhythmias: an overview. Heart Rhythm. 2007;4(7):964-72.

9. Castro Hevia J, Antzelevitch C, Tornes Barzaga F, Dorantes Sanchez M, Dorticos Balea F, Zayas Molina R, et al. Tpeak-Tend and Tpeak-Tend dispersion as risk factors for ventricular tachycardia/ ventricular fibrillation in patients with the Brugada syndrome. $J$ Am Coll Cardiol. 2006;47(9):1828-34.

10. Erikssen G, Liestol K, Gullestad L, Haugaa KH, Bendz B, Amlie JP. The terminal part of the QT interval (T peak to $\mathrm{T}$ end): a predictor of mortality after acute myocardial infarction. Ann Noninvasive Electrocardiol. 2012;17(2):85-94.

11. Smetana P, Schmidt A, Zabel M, Hnatkova K, Franz M, Huber K, et al. Assessment of repolarization heterogeneity for prediction of mortality in cardiovascular disease: peak to the end of the $\mathrm{T}$ wave interval and nondipolar repolarization components. J Electrocardiol. 2011;44(3):301-8.

12. Haarmark C, Hansen PR, Vedel-Larsen E, Pedersen SH, Graff C, Andersen MP, et al. The prognostic value of the Tpeak-Tend interval in patients undergoing primary percutaneous coronary intervention for ST-segment elevation myocardial infarction. J Electrocardiol. 2009; 42(6):555-60.

13. Watanabe N, Kobayashi Y, Tanno K, Miyoshi F, Asano T, Kawamura $\mathrm{M}$, et al. Transmural dispersion of repolarization and ventricular tachyarrhythmias. J Electrocardiol. 2004;37(3):191-200.

14. Morin DP, Saad MN, Shams OF, Owen JS, Xue JQ, Abi-Samra FM, et al. Relationships between the T-peak to T-end interval, ventricular tachyarrhythmia, and death in left ventricular systolic dysfunction. Europace. 2012;14(8):1172-9.
15. Gupta P, Patel C, Patel H, Narayanaswamy S, Malhotra B, Green $\mathrm{JT}$, et al. $\mathrm{T}(\mathrm{p}-\mathrm{e}) / \mathrm{QT}$ ratio as an index of arrhythmogenesis. $J$ Electrocardiol. 2008;41(6):567-74.

16. Dodge JT, Jr., Brown BG, Bolson EL, Dodge HT. Intrathoracic spatial location of specified coronary segments on the normal human heart. Applications in quantitative arteriography, assessment of regional risk and contraction, and anatomic display. Circulation. 1988;78(5 Pt 1):1167-80.

17. Yamaguchi M, Shimizu M, Ino H, Terai H, Uchiyama K, Oe K, et $a l$. T wave peak-to-end interval and QT dispersion in acquired long QT syndrome: a new index for arrhythmogenicity. Clin Sci (Lond). 2003;105(6):671-6.

18. Gibson CM, Cannon CP, Daley WL, Dodge JT, Jr., Alexander B, Jr., Marble SJ, et al. TIMI frame count: a quantitative method of assessing coronary artery flow. Circulation. 1996;93(5):879-88.

19. Letsas KP, Weber R, Astheimer K, Kalusche D, Arentz T. TpeakTend interval and Tpeak-Tend/QT ratio as markers of ventricular tachycardia inducibility in subjects with Brugada ECG phenotype. Europace. 2010;12(2):271-4.

20. Beltrame JF, Turner SP, Horowitz JD. Persistence of the coronary slow flow phenomenon. Am J Cardiol. 2001;88(8):938.

21. Kurtoglu N, Akcay A, Dindar I. Usefulness of oral dipyridamole therapy for angiographic slow coronary artery flow. Am J Cardiol. 2001;87(6):777-9, A8

22. Tambe AA, Demany MA, Zimmerman HA, Mascarenhas E. Angina pectoris and slow flow velocity of dye in coronary arteries--a new angiographic finding. Am Heart J. 1972;84(1):66-71.

23. Mosseri M, Yarom R, Gotsman MS, Hasin Y. Histologic evidence for small-vessel coronary artery disease in patients with angina pectoris and patent large coronary arteries. Circulation. 1986;74(5):964-72.

24. Yaymaci B, Dagdelen S, Bozbuga N, Demirkol O, Say B, Guzelmeric $\mathrm{F}$, et al. The response of the myocardial metabolism to atrial pacing in patients with coronary slow flow. Int J Cardiol. 2001;78(2):151-6. 